\title{
Deshojando margaritas: ¿nanociencia o nanotecnología?
}

\author{
Ernesto Estévez Ramos* \\ Beatriz Aragón Fernández**
}

Toda ciencia reúne en sí la capacidad potencial de transformar el mundo. Al conocimiento científico nuevo le es añadido, aún si es sólo en carácter de posibilidad, la capacidad de ser utilizado para transformar la realidad. Quizás el caso más visible en que ese poder transformador se realiza, sea cuando ocurre en forma de tecnología. Hay en la relación entre ciencia y tecnología una tensión latente, de seguro dialéctica, que en el mundo contemporáneo tiene solución a diferentes niveles y que se proyecta de manera marcada a la sociedad como un todo. Si en el pasado la capacidad tecnológica y por tanto, transformadora de la ciencia se daba a escala local y su expansión a ámbitos más globales se lograba en un lapso extendido de tiempo, en el mundo contemporáneo ciencia y tecnología van de tal punto juntos que su sinergia puede provocar, para bien o para mal, consecuencias globales en intervalos asombrosamente cortos de tiempo.

En el instante en que ciencia y tecnología alcanzan ese poder revolucionador, ya han pasado suficientes siglos de organización social del trabajo para llegar a la transformación de la ciencia y la tecnología de sucesos individuales, a elementos sociales influidos e influyentes en la estructura de la sociedad y su dinámica. El análisis de la relación entre ciencia y tecnología deja entonces de ser un tema de tertulia contemplativa, para tornarse una necesidad a fin de entender la lógica del mundo actual.

La nanociencia y la nanotecnología son resultado del desarrollo científico técnico que ha permitido al hombre manipular la materia a la escala de una millonésima de milímetro, la escala del nanómetro. Sólo para entender la pequeñez de esta escala: si infláramos el universo de manera tal, que una esfera originalmente del tamaño de un nanómetro terminara siendo de un metro de diámetro, una canica de jugar bolas se habría expandido hasta ser del tamaño del planeta tierra.

Esta área del conocimiento se anuncia como la próxima revolución civilizatoria del hombre que ya hoy está en plena marcha. La capacidad de conocer y trabajar los materiales a esa escala, permite actuar de manera directa sobre la naturaleza misma de lo material, sin distinción entre lo biológico y lo inanimado. Las consecuencias de esta revolución apenas pueden ser calculadas hoy en toda sus dimensiones, pero está claro ya, que afectarán de manera dramática al hombre y a la naturaleza. ${ }^{1}$

En el caso de las nanociencias y las nanotecnologías, ya es lugar común englobar a ambas por el último nombre, insistiéndose en la inseparabilidad práctica de ambos

\footnotetext{
* Instituto de Ciencia y Tecnología de Materiales, Universidad de la Habana, Cuba.

** Universidad de la Ciencias Informáticas, Cuba.

1 Para una explicación más detallada sobre las nanociencia y las nanotecnología, el lector puede consultar “Las nanotecnologías en un mundo disfuncional", de Ernesto Estévez y Beatriz Aragón, en Rebelión: <http:// www.rebelion.org/noticia.php?id=89212, 2009>.
} 
términos. En este discurso se argumenta que la intencionalidad práctica de la investigación básica y el acortamiento del ciclo investigación-desarrollo-impacto social ha borrado las fronteras entre la ciencia y la tecnología, desarrollándose ambas de manera simultánea y en una interacción indisoluble. Contrario a esto, también se desarrolla una línea argumental que quiere separar en términos concretos las nanociencias de las nanotecnologías. En ella, reconociéndose la dificultad práctica, se insiste de todos modos en darle valor real a la diferencia conceptual entre ambas.

La discusión no es fútil. Detrás de este debate pueden esconderse intereses no tan académicos y neutrales, con una intencionalidad ideológica y económica bien definida. Al discurso que lo engloba todo en nanotecnología no le es ajena la pretensión de captar recursos frescos sobre la base de que el conocimiento que se genera en los laboratorios tiene un valor de mercado alto y realizable. Un segundo discurso, que intenta separar la relación nanociencia-nanotécnología, se hace sospechoso cuando se le dicta a los investigadores e instituciones del tercer mundo para insistir en la apertura de sus investigaciones, su inserción en proyectos de investigación del primer mundo y otras formas de manipulación.

En el caso de Cuba, aún cuando el contexto nacional difiera de su entorno tercer mundista, esta discusión tampoco es bizantina. En un momento en que el país está definiendo una estrategia sobre su desarrollo en el área de las nanotecnologías, debates sobre estos asuntos no pueden ser subestimados en la conformación de un consenso entre los diversos actores y públicos relacionados con las nano y en la decisión de un curso de acción determinado.

El presente texto discute si es correcto (y real) separar nanociencia de nanotecnología y la relevancia de esta discusión para entender el fenómeno nano en varias de sus implicaciones. Si en un texto anterior se argumentaba sobre la incidencia de la revolución nano sobre el mundo actual, aquí, dentro de la línea argumental principal, el énfasis se hará en la incidencia de ese contexto sobre el propio desarrollo de las nano.

La lógica del discurso que seguiremos no es del todo lineal, comenzaremos por discutir sobre la distinción entre nanociencia y nanotecnología para luego ampliar el marco de la discusión. Se valorará el contexto de los países periféricos y cómo la disyuntiva entre nanociencia y nanotecnología es parte de un argumento más general, que ha de tener en cuenta la realidad social en que esta revolución está teniendo lugar. Esperamos que al final de la lectura se entienda que lo nano sólo puede ser abordado desde una perspectiva inclusiva de las ciencias naturales y sociales.

\section{DE CÓMO SE LLEGÓ DE UN DESCUBRIMIENTO FísICO A LOGRAR "OJOS" ÍNFIMOS PARA HURGAR MEMORIAS}

En 1988, se descubrió el fenómeno de la magnetorresistencia gigante que permitió, diez años después, fabricar las cabezas lectoras que abrieron las puertas a los discos duros de más de un gigabyte, tan comunes hoy en las computadoras modernas (1 gigabyte es equivalente a 8,589,934,592 respuestas de sí o no).

La magnetorresistencia gigante es una propiedad relativamente fácil de explicar. Ocurre cuando un material es capaz de cambiar su resistencia eléctrica en mucho, cuando el campo magnético bajo el cual se encuentra cambia muy poco. Como en un disco duro, la información se almacena en "trocitos" virtuales de imanes, entonces, un 
sensor basado en el fenómeno de la magnetorresistencia gigante puede "ver" unidades de información muy pequeñas, permitiendo aumentar drásticamente la cantidad de información almacenada en el mismo volumen.

En 1997, la compañía IBM anunció el primer cabezal magnetorresistente y el primer disco duro de 17 gigabytes. Hoy en día, utilizando cabezales basados en el mismo fenómeno, los discos duros alcanzan una capacidad de 2 terabyte $(175,921,860,444$ respuestas de sí o no). Si en los primeros treinta años el hombre había logrado multiplicar la capacidad de los discos duros en 47 veces, de 5 Mbytes a 236 Mbytes en 1988, en los próximos veinte, el salto sería por un factor de más de 8,000 veces.

En el año 2007, Peter Gruenberg y Albert Fert obtuvieron el premio Nobel de Física por el descubrimiento de la magnetorresistencia gigante.

\section{Los materiales que muestran este efecto son materiales nanoestructurados}

En esta historia hay varias cosas que llaman la atención: el tiempo breve que medió entre el descubrimiento del fenómeno físico y su aplicación comercial. Este tiempo es cinco veces menor que el transcurrido desde los primeros experimentos eléctricos y el primer bombillo incandescente comercial.

Por otro lado, está la intencionalidad de la investigación básica. Si los pioneros investigadores de la electricidad lo hacían por pura curiosidad intelectual, sin avizorar las posibles aplicaciones, en el caso de la magnetorresistencia gigante, el fenómeno "gritaba" sus aplicaciones desde el mismo inicio. En ese sentido podríamos decir que el gérmen tecnnológico ya existía en el descubrimiento básico.

\section{AQUí SE INTENTA RESPONDER LA PREGUNTA DE LOS VEINTE PESOS: ¿Pueden separarse nanociencia y nanotecnología?}

La nanociencia se refiere a la investigación en la escala nanométrica que tiene como fin la sistematización de conocimiento con independencia de su utilidad práctica. Es decir, la investigación que busca el descubrimiento y comprensión de fenómenos en la nanoescala, la acumulación estructurada de conocimiento nuevo, la conceptualización de nuevas categorías, sus relaciones y leyes.

La nanotecnología es el desarrollo de procesos, procedimientos y productos capaces de llevar el conocimiento de la nanoescala a aplicaciones prácticas, cuyas implicaciones han de ir, directa o indirectamente, más allá del laboratorio o centro que los produce para impactar a la sociedad de algún modo.

El descubrimiento de la magnetorresistencia gigante es nanociencia, el diseño y construcción de cabezales magnéticos basados en ello es nanotecnología.

Pero aún cuando pudiera pensarse que nanociencia y nanotecnología son separables, un análisis apenas más profundo revela que en el sentido práctico es casi imposible lograrlo.

Se pueden enumerar varias razones para argumentar esta última afirmación:

1) La investigación en la nanoescala necesita en muchos casos del desarrollo ad hoc, a nivel de laboratorio, de instrumentación científica que, en muchas ocasiones, termina siendo un producto comercializable. 
La nanociencia está, desde el punto de vista instrumental, en una etapa de desarrollo agresivo. Esto la diferencia de otras ciencias con más tradición, donde el "paquete estandard" de instrumentos científicos se halla bien establecido comercialmente. En nanociencia una parte importante de la investigación viene acompañada por el desarrollo o modificación de los instrumentos que se utilizan en el laboratorio.

Entre 1982 y 1985, Rohr, Berning y Gerber inventaron el microscopio de efecto túnel y el microscopio de fuerza atómica, los dos representantes fundamentales de una familia de instrumentos de observación, análisis y manipulación de superficies que hoy agrupa a más de una docena de instrumentos. Tal es el ímpetu en el desarrollo de estos instrumentos que hay hasta una revista científica, Journal of Scanning Probe Microscopy, dedicada exclusivamente a recoger estos resultados. La mayoría de estos instrumentos son desarrollos realizados en los laboratorios de investigación y algunos de ellos han saltado de los laboratorios a empresas que los producen y comercializan. Se puede establecer una cronología muy ilustrativa entre hitos en el desarrollo de los principales instrumentos de microscopía utilizados en lo nano, y la creación de empresas surgidas de laboratorios universitarios.

Incluso aquellos desarrollos instrumentales que no llegan a la escala industrial pueden ser comercializados directamente por los propios laboratorios como productos para otros laboratorios de nanotecnología. El alto valor agregado de muchos de estos instrumentos implica un ingreso económico importante.

TABLA 1. Hitos en el desarrollo de microscopios de superficies y hechos académicoempresariales.

\begin{tabular}{|c|c|c|}
\hline Hito instrumental & Año & Hecho académico-empresarial \\
\hline Microscopio de efecto túnel (STM). & 1982 & \\
\hline Microscopio de fuerza atómica (AFM). & 1985 & $\begin{array}{l}\text { Investigadores de la Universidad de Stanford } \\
\text { crean la empresa Park Scientific. }\end{array}$ \\
\hline Microscopio óptico de barrido (NOM). & 1984 & \\
\hline Microscopio de fuerza atómica (MFM). & 1987 & $\begin{array}{l}\text { Investigadores de la Universidad de California } \\
\text { crean Digital Instruments. }\end{array}$ \\
\hline $\begin{array}{l}\text { Se crea el primer instrumento de AFM con } \\
\text { Tapping Mode. }\end{array}$ & 1993 & $\begin{array}{l}\text { Molecular Imaging es fundada por investiga- } \\
\text { dores de la Universidad Estatal de Arizona. }\end{array}$ \\
\hline- & 1997 & $\begin{array}{l}\text { Investigadores de la Universidad de Basel fun- } \\
\text { dan Nanosurf. WITec Es creada por investiga- } \\
\text { dores de la Universidad de Ulm }\end{array}$ \\
\hline
\end{tabular}

2) El objeto de estudio en la nanociencia es, en la mayoría de los casos, artificial. Eso que implica la necesidad de diseñar métodos de obtención que constituyen en sí mismo tecnologías o gérmenes de ella. Más aún, si bien el descubrimiento puede ser resultado de la curiosidad científica, su potencial como tecnología puede escapar a la intención original de los investigadores.

En 1985, Smalley, Curl y Kroto, buscando sintetizar en el laboratorio el polvo intergaláctico, descubrieron los fullerenos, una nueva forma estable de carbono que les valió el premio Nobel de Química en 1996. Ya en el 2003, se producía más de 40 toneladas de ese producto al año. En el 2008, el mercado global de fullerenos alcanzó 
los 300 millones de USD y se predice que para 2015 habrá alcanzado los 4,600 millones de USD. ${ }^{2}$

No es el único ejemplo

En octubre del 2009, el profesor Ian W. Boyd fue designado al frente del Centro de Melbourne para Nanofabricación ${ }^{3}$ cuya misión es "proveer instrumentación y facilidades para la fabricación de dispositivos que puedan potencialmente revolucionar muchos aspectos de la industria". El profesor, adscrito al University College of London, era jefe del grupo de materiales y dispositivos, que se dedica a investigaciones fundamentales para la obtención de materiales en películas delgadas. La experiencia en investigaciones básicas del Dr. Ian W. Boyd es, sin dudas, muy apreciada por quienes lo nombraron al frente de un centro cuyo propósito es explícitamente tecnológico.

Otro ejemplo es el del mercado de las nanopartículas; se espera que alcance en el 2009 los 40 mil millones de USD. La inmensa mayoría de estos productos que se comercializan tienen su origen en investigaciones básicas. La búsqueda de nuevos materiales tiene como requisito previo buscar la forma de obtenerlos, no importa si es por simple interés científico o por interés comercial.

3) El proceso de investigación genera en muchos casos, casi de inmediato y de manera evidente, una intencionalidad tecnológica, aun cuando ésta no se realice en el mismo lugar donde se desarrolla la investigación básica.

Un ejemplo de ciencia básica que grita su aplicación es el resultado obtenido entre la Universidad de Yale y el Instituto Nacional de Standards de los EUA (NIST), que, investigando los mecanismos eléctricos en las células, han inventado células sintéticas en los que se crea una batería eléctrica de la que se puede obtener una diferencia de voltaje utilizable: "una pequeña batería de sólo 200 nanolitros permitiría obtener electricidad por cerca de 10 minutos". Los investigadores creen que este resultado abre el camino para suministrar energía a nanodispositivos. ${ }^{4}$

Quizá sea por esa potencialidad que tiene la nanociencia de tornarse nanotecnología de manera casi espontánea, que al físico del Massachusetts Institute of Technology, Pablo Jarillo, le han otorgado un premio de 875,000 USD para investigaciones básicas en las propiedades de los grafenos. ${ }^{5}$ El fondo ha sido otorgado por la fundación David y Lucile Packard, la organización "no lucrativa" creada por el fundador de la empresa de alta tecnología Hewlett-Packard.

El hecho de que un laboratorio no realice una investigación con el objetivo de aplicarlo, no califica su trabajo como ciencia básica. La realidad es que otros pueden apropiarse, explícitamente o no, del resultado, y tornarlo un producto vendible. El ciclo de investigación-desarrollo-impacto social se ha vuelto tan corto que la delimitación de

\footnotetext{
2 The Global Market for Carbon Nanotubes and Fullerenes. Technology Transfer Centre Ltd. Febrero de 2009.

3 London Centre for Nanotechnology Professor to Lead Melbourne Centre for Nanofabrication. <http://www. azonano.com/news.asp?NewsID=13834>, 2009.

4 Artificial Cells Acting as a Tiny Battery. <http://www.azonano.com/news.asp?newsID=14241>, 2009.

5 Five year grant allows MIT physicist to explore unique features of graphene. <http://www.azonano.com/ news.asp?newsID=14178>, 2009.
} 
cada uno de sus componentes se vuelve confuso. No por gusto, en los países desarrollados, cerca del 85\% de las multinacionales activas en nanotecnologías tienen alianzas y colaboraciones formales con universidades y laboratorios financiándole parcial o totalmente sus investigaciones básicas. ${ }^{6}$

Las compañías y gobiernos entienden muy bien el valor tecnológico de lo básico, como explica el profesor Alex Callinicos cuando expresa, respecto a las universidades británicas, que éstas "están reconstruyéndose para ofrecer a las corporaciones británicas y extranjeras la investigación académica y los trabajadores cualificados que necesitan para ser rentables". ${ }^{7}$ El potencial tecnológico surge en los laboratorios universitarios pero se realiza en las corporaciones. En el caso del mundo desarrollado, todavía existe la posibilidad de que ese potencial se logre concretar en el propio país. En el caso del tercer mundo, la realidad es más cruda. La investigación básica que se realiza en los laboratorios universitarios, en la mayoría de los casos termina siendo aprovechada fuera de sus fronteras, en el primer mundo, donde se tienen las condiciones de infraestructura para llevar el germen tecnológico creado en el país pobre a un producto comercializable. Las maneras en que este fenómeno se da han sido analizadas con anterioridad. ${ }^{8,9}$

4) El alto valor agregado, unido a los pequeños volúmenes requeridos, de algunos productos obtenidos en el proceso de investigación hace posible su comercialización directa sin necesidad de escalado. Existe un mercado de comercialización de productos que no implica la creación de un proceso productivo de escala industrial.

En este tema ocurre algo similar a la instrumentación. Los laboratorios comercializan de manera directa pequeñas cantidades de nanobjetos, de alto valor agregado, que producen en sus áreas de investigación básica, con mayor ventaja cuando son dueños exclusivos del conocimiento para producirlos.

El profesor Kattesh Katti, de la Universidad de Missouri, ha desarrollado un método que reduce el tiempo de síntesis de nanopartículas de oro y plata de unas 30 horas a varios minutos. El propio profesor explica que su laboratorio será capaz de suministrar las nanopartículas a otros laboratorios nano que lo necesiten, "ciertamente seremos capaces de suministrar y ayudar a investigadores en todos los Estados Unidos". ${ }^{10}$

Más aún, hay laboratorios de investigación básica que ofertan el servicio de síntesis de materiales o dispositivos al pedido, en cantidades pequeñas a precios que pueden resultar exorbitantes. En este caso, la nanotecnología, indistinguible de la nanociencia, es el desarrollo de métodos generales de síntesis o fabricación de nano "cosas". Métodos adaptables y flexibles para un mercado que solicita de acuerdo con sus necesidades y no de acuerdo con lo disponible

\footnotetext{
${ }^{6}$ Gian Carlo Delgado-Ramos. 2008. Guerra por lo invisible: negocio, implicaciones y riesgos de la nanotecnología. Colección El Mundo Actual. México: CEIICH-UNAM: 114.

7 Callinicos, Alex. 2006. Universities in a neoliberal world. Londres. Bookmarks Publications.

8 Delgado-Ramos, Gian Carlo. 2008. Guerra por lo..., op. cit.; 221.

9 Estévez, Ernesto. "La fábula de los tres hermanos: las nanociencias y las nanotecnologías en el contexto cubano". Revista Temas (en proceso de publicación).

${ }^{10}$ Nanoparticles to aid medical diagnosis, therapy. <http://www.innovations-report.com/html/reports/life_ sciences/report-36440.html>, 2004.
} 
La compañía alemana MicroParticle fue fundada en 1997 por un grupo de científicos de la Academia de Ciencias de Berlín para comercializar nanopartículas poliméricas monodispersas. Basados en los métodos desarrollados en sus laboratorios de origen, hoy tiene entre sus principales clientes universidades, laboratorios y sectores industriales. ${ }^{11}$

La industria del espionaje y la guerra suele ser un cliente recurrente de este tipo de servicio, donde en ocasiones se necesita un producto en pequeñas cantidades o único, una sola vez. Sectores de alta tecnología también suelen alimentar este mercado: las agencias espaciales como la NASA, grandes laboratorios o facilidades experimentales nacionales como el Centro Europeo de Investigaciones Nucleares (CERN) el NIST de los Estados Unidos etc. Lo importante de este sector es que, aún cuando los pedidos pueden ser únicos, el precio del servicio puede llegar a los millones. A la sombra de esta realidad es que se ven crecer compañías cuyo mercado son precisamente estos grandes contratistas exclusivos.

5) Los laboratorios dedican una parte de sus esfuerzos al desarrollo y ejecución de servicios altamente especializados para la industria, como resultado intrínseco, directo o muchas veces como subproducto de sus investigaciones en nanociencias.

Esta realidad no es nueva, ni coto exclusivo de las nanotecnologías, y es un segmento de mercado que se torna importante en un contexto de penetración neoliberal de las universidades. Los institutos de investigación dentro de las universidades se especializan en realizar labores de expertos para la industria, redituables financieramente. Los servicios pueden ser muy variados, desde la opinión experta sobre la viabilidad de una propuesta, hasta el desarrollo de materiales al pedido.

Esto lo explican de la siguiente manera:

Desde principios del siglo XX, las grandes compañías tendieron a hacer investigaciones en sus propios laboratorios: esto fue cierto, por ejemplo, en el caso de la industria química alemana y en los laboratorios Bell en Estados Unidos de América. En todo caso, esto está cambiando. Ahora los productos son tan complejos, que se requiere investigación en una gama de técnicas más amplia de las que cualquier compañía puede asumir. [...] En este entorno cambiante, las universidades son socios potencialmente muy atractivos para el mundo de los negocios. Los buenos investigadores universitarios funcionan en redes internacionales: saben en qué lugar del mundo se hacen trabajos punteros de su campo. [...] los laboratorios universitarios se refrescan continuamente con la llegada de nuevos investigadores inteligentes en forma de estudiantes posgraduados y profesores. ${ }^{12}$

Cuando una compañía transnacional tuvo interés en desarrollar un material magnético nanoestructurado, se acercó al grupo del Prof. Josef Fidler de la Universidad Técnica de Viena, que puso a su disposición las herramientas que habían desarrollado durante años de trabajo en nanomagnetismo para este proyecto tecnológico. Su experticia en temas de ciencia básica fue clave en lograr la meta en menos de seis meses de trabajo. ${ }^{13}$

\footnotetext{
${ }^{11}$ MicroParticle <http://www.microparticles.de/micropartengl.html>, 2009.

${ }^{12}$ Lambert Review of Business-University Collaboration: Final Report, 2003.

${ }^{13}$ Comunicación privada con el Prof. Josef Fidler y colaboradores, 2009.
} 
En un sentido más general, el conocimiento acumulado se torna un activo comercializable en forma de producto intangible. Las universidades se vuelven espacios universalizadores de cierto tipo de conocimiento que el sector productivo no puede prever ni mantener. A ella se recurre cuando se necesita de una experticia que puede desbordar un área particular, tiene la flexibilidad de lograr integrar en sus análisis investigadores muy diversos y dar un enfoque más completo. La ausencia por lo general de un entorno crispado por las presiones productivas y el balance de ganancia, les permite desarrollar herramientas más generales, pensar en contextos más amplios, buscar un conocimiento más estratégico y avizorar caminos más arriesgados de investigación.

6) La apropiación legal del conocimiento logrado desde las etapas iniciales de la investigación va más allá de patentar procesos y productos, y alcanza la protección excluyente de conocimiento básico y fundamental.

Esta realidad ha sido analizada desde diferentes puntos de vista. ${ }^{14}$ Las patentes de conocimiento básico abundan. En el caso de la nanotecnología, la explosión de ellas ya es una realidad. Un ejemplo de esta tendencia ya ha sido analizado: la capacidad de la salamandra de adherirse a superficies muy lisas es resultado de protuberancias nanométricas en las plantas de sus extremidades. Este resultado, esencialmente básico, ha sido protegido con varias patentes. Está lejos de ser el único ejemplo.

Que la transferencia de conocimiento básico constituye un activo altamente cotizado está siendo descubierto por las universidades. En julio del 2006, el Imperial College, del Reino Unido, fue la primera universidad en hacer pública su oficina de transferencia técnica de propiedad universitaria, cotizándose en más de 25 millones en bolsa tan sólo con el 14\% de sus activos. ${ }^{15}$ De acuerdo con el analista J. Boone, del Financial Times, las compañías de comercialización de la propiedad intelectual están en competencia feroz por ver quienes firman acuerdos de exclusividad con las principales universidades británicas. ${ }^{16}$

En junio del 2007, la compañía nanoTerra compró los derechos de licencia de más de 50 patentes a la Universidad de Harvard..$^{17}$ Las patentes cubren técnicas para el diseño de materiales que se autoensamblan, herramientas para elaborar circuitos en la nanoescala, lentes y dispositivos de memorias, entre otros. NanoTerra anunció que ya ha firmado acuerdos con el Departamento de Defensa de los EUA, la compañía alemana Merk y el gigante de la química 3M.

Que la selva de patentes ya constituye un obstáculo para el desarrollo de las nanos es una realidad. En el área de los nanotubos de carbono se estiman en unas 543, solamente en Estados Unidos y que cubren cada paso en el proceso de fabricación y utilización de estos materiales.

Fabricantes de productos de nanotubos de carbono, que no desean depender de suministradores externos para sus productos, se enfrentan a problemas de patentes en todas las etapas

\footnotetext{
${ }^{14}$ Pérez, Patricia. 2006. "Patentes limitan uso de nanotecnología", Science and Development Network, <www. scidev.net/en/science-and-innovation-policy/education/news/patents-limit-use-of-nanotechnology. html>, 2006.

${ }^{15}$ Boone, J. 2006. "University company sells stake for 25 million pounds". Financial Times.

${ }^{16}$ Boone, J. 2006. "Most colleges set to sign technology transfer deals", Financial Times.

17 Bullis, K. 2007. "Key nanotech patents licensed”. <http://technologyreview.com/biomedicine/18829/page1/>.
} 
de sus síntesis: las técnicas de crecimiento (descarga de arco, deposición láser, deposición química en fase de vapor); las herramientas de procesamiento de los nanotubos (para la purificación, ordenamiento, dopaje, funcionalización, recubrimiento); las técnicas de manipulación para su deposición final o su integración en el producto. ${ }^{18}$

FIGURA 1. Número acumulativo de patentes en nanotecnologías otrogadas por el USPTO (modificado de: The patent land grab in nanotechnology continues unabated, creating problems down the road. $<$ http://www.nanowerk.com/spotlight/spotid=386.php >).

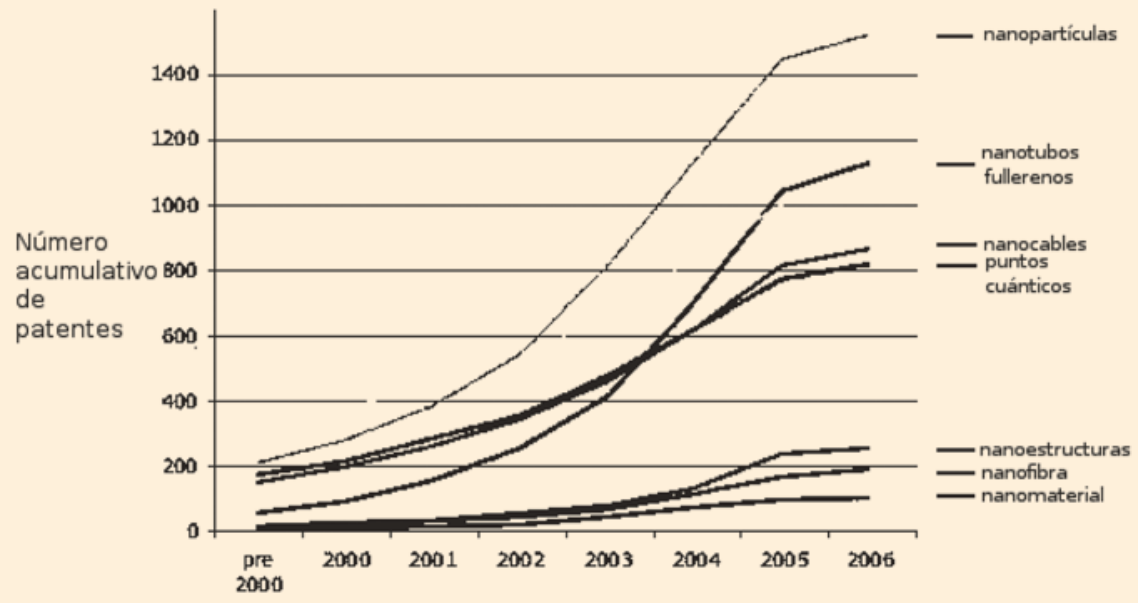

Pero el problema no se reduce a los nanotubos de carbono. El número de patentes nano crece a ritmo muy rápido anualmente como muestra la figura 1 y una buena parte de las otorgadas es sobre aspectos básicos como procesos de síntesis, manipulación, entre otros.

En el contexto de la apropiación del conocimiento, separar nanociencia y nanotecnología no es ya un mero error conceptual, es un error estratégico, pues se tiene la amenaza muy real de que no sólo los caminos a las aplicaciones se cierren, sino de que estas puertas cerradas también impidan el acceso a la búsqueda de conocimiento y a las rutas que de él parten.

La inseparabilidad de la ciencia y la tecnología en el área nano ha llevado a utilizar el término nanotecnología para englobar a ambas. No creemos afortunado tal uso y preferimos hablar de lo "nano" para sintetizar su carácter indisoluble.

\section{AHORA SE INTRODUCEN OtRAS PREGUNTAS RESULTAdO DE LA ANTERIOR Y SE JUSTIFICA QUE SON TAN IMPORTANTES COMO LA INTERROGANTE ORIGINAL}

En algunos discursos públicos se intenta escamotear que surjan preguntas de fondo sobre el tema de cómo abordar el tema nano en las políticas científicas y de desarrollo de los países pobres. Partiendo de que la revolución nano se presenta como la

\footnotetext{
18 "Growing nanotechnology problems: navigating the patent laberynth". Nanowek. <http://www.nanowerk. com/spotlight/spotid=1367.php>. 2007.
} 
nueva piedra filosofal que resolverá todos los problemas de la sociedad se arguye que para lograr tal fin, los países subdesarrollados deben "integrar" sus iniciativas en este campo al de las potencias mundiales. Frente a este discurso hay que preguntarse qué peligros acechan detrás de estos aparentes llamados altruistas a la integración asimétrica, y qué dinámica deben seguir las iniciativas nano de los países en desarrollo.

La vinculación en el mundo subdesarrollado de los proyectos nano nacionales, universitarios o incluso individuales, con los centros de poder internacionales provoca que, en no pocas ocasiones, las investigaciones no tengan nada que ver con el contexto nacional donde se desarrollan. En el 2007, se desarrolló, en México, la reunión NanoforumEULA para tratar las investigaciones de nanomateriales en América Latina y en Europa. Como resultado de ello se decidió crear un fondo de 20 millones de euros para fomentar proyectos conjuntos de nanotecnologías México-UE. Uno de los objetivos declarados de la iniciativa es "apoyar a las empresas que usan las nanos dentro de sus procesos para mejorar su competitividad"19. Uno no puede menos que preguntarse, apoyar empresas de dónde, de México o de Europa? ¿La competitividad de quién, de Mexico o de Europa? Estas dudas han sido abordadas por el investigador mexicano Guillermo Foladori, quien ha señalado que "el gobierno federal (mexicano) ha firmado acuerdos con centros de investigación, universidades extranjeras e industrias para promover el desarrollo de la ciencia diminuta, por lo cual todo producto quedará fuera del control del país". ${ }^{20}$

El problema no se reduce a los gobiernos. Como afirma Foladori “la opinión de los científicos que trabajan lo nano no necesariamente coincide con los caminos que la gente considera apropiados para satisfacer sus necesidades". ${ }^{21}$ La pretensión falsa de separar nanociencia de nanotecnología en ocasiones es utilizada para justificar el posicionamiento social del investigador.

Desde una aparente distinción de que se trata solamente de ciencia, sobre los actores individuales y colectivos inciden presiones, como la de publicar en revistas científicas internacionales (en su amplia mayoría pertenecientes a conglomerados editoriales transnacionales) para lograr reconocimiento, acceder a mejores salarios en sus universidades, o aspirar a categorías laborales superiores. Esta presión real, conlleva a emprender investigaciones en áreas calientes de las nano, en muchos casos calentadas precisamente desde el contexto primer mundista, para lograr publicar en revistas de alto prestigio, elevar el estatus frente a sus pares y lograr becas y subsidios del primer mundo. Poco importa si el tema de investigación, presentada exclusivamente como ciencia, termina siendo útil (como lo que realmente es, tecnociencia) para una empresa monopolista de cosméticos, en vez de tributar a la solución de un problema de salud local. Esta tendencia se ve reforzada por la estructura de estímulos implementados en muchas universidades del tercer mundo, que son copia mimética de los de las universidades europeas o norteamericanas.

Debe quedar claro que no se trata de invalidar emprendimientos en áreas punteras, aún si están lejos de la necesidad inmediata. Se trata de que estas investigaciones

\footnotetext{
19 "México y Europa colaborarán en nanotecnología". Milenio. <http://impreso.milenio.com/node/7110695>. 2008.

${ }^{20}$ Laguna, Mauricio. 2007. “México, dependiente de nanotecnología”. Revista Fortuna. , <http://revistafortuna. com.mx/opciones/archivo/2007/septiembre/htm/mexico_nanotecnologia.htm>.

${ }^{21}$ Invernizzi, Noela y Guillermo Foladori. 2008. ¿Serán las nanotecnologías una solución a la pobreza en el mundo? Capítulo 1 en nanotecnología en la agricultura y la alimentación. CSEAM.
} 
se enfocan con un fin de ventaja individual inmediata. El objetivo del proyecto de investigación termina siendo publicar bien y recoger los frutos de esa cosecha, sin insertar la investigación en un proyecto colectivo coherente, de mayor trascendencia para el contexto en que se viven y se desarrollan los actores de la investigación. Es el síndrome de investigar para Nature ${ }^{22}$ y para la IBM. Las consecuencias sociales de este fenómeno no pueden despreciarse. El actor científico se enajena de su realidad inmediata, conviviendo con sus connacionales pero sintiéndose que pertenece a otro colectivo, supranacional, exclusivo, de otros actores como él, que publican donde él publica, que lo entienden y a quienes en última instancia les debe lealtad. Toda una mentalidad colonial disfrazada de modernidad y globalización aideológica, sin percatarse que, como individuo, está siendo utilizado como una pieza desechable de una maquinaria perversa de reproducción social.

Tal actitud de alienación tiene también causas endógenas. Un investigador o colectivo mal comprendido, no reconocido o apoyado en su contexto, es un científico que se deja a merced del acecho de otros depredadores sociales. Tal es la causa en no pocas ocasiones, del actor que termina siendo financiado directa o indirectamente, sin vínculo efectivo local, por instituciones allende al patio y por tanto termina insertándose en las dinámicas de investigación+desarrollo+impacto social de otras geografías que no son las propias. Si bien la fuga de cerebros tiene como componente fundamental los cantos de sirenas de otras entidades con ofertas materiales más tentadoras, también es cierto que la falta local de canto alguno (perspectiva y proyecto de desarrollo local), el mal diseño de las políticas de investigación e innovación, la falta de reconocimiento, y ser relegado al pretérito cuando se habla de nuevas iniciativas, todas actúan como causas propias de este fenómeno global. En todo caso, el actor que localmente fue despreciado, resulta que si es apreciado en el mismo norte que aparentemente tiene menos necesidad de él, que el sur de donde se ha ido.

\section{Dónde SE HACE UNA PAUSA REFLEXIVA PARA SEGUIR ARGUMENTANDO SOBRE EL CONTEXTO SOCIAL}

Varias décadas antes de que lo nano se volviera un término popular, investigaciones que hoy clasificaríamos como tales, se realizaban en varios laboratorios del mundo. Lo que hoy se llama con soltura nanociencia y nanotecnología no está claramente delimitado y es incluso motivo de controversia. Para dar sentido a esta realidad hay que entender que lo que distingue el contexto actual es nuestra habilidad de hacer tecnociencia en la escala nano con un impacto social masivo. Tecnociencia es un "recurso del lenguaje para denotar la íntima conexión entre ciencia y tecnología y el desdibujamiento de sus límites". ${ }^{3}$

Richard W. Siegel, del Consejo Nacional de Ciencia y Tecnología de los EUA, lo explica del siguiente modo. ${ }^{24}$

\footnotetext{
${ }^{22}$ Nature es una de las dos revista de ciencias más prestigiosa del mundo perteneciente a la empresa editorial McMillian.

${ }^{23}$ Nuñez Jover, Jorge. 2002. “De la ciencia a la tecnociencia: pongamos los conceptos en orden”. Tomado de $L a$ ciencia y la tecnología como procesos sociales, La Habana: Editorial Félix Varela.

${ }^{24}$ Siegel, R. W. 1999. Nanostructure science and technology. A worldwide study. Capítulo 1. National Science and Technology Council (NSTC).
} 
Mientras muchos aspectos de este campo existían mucho antes de que la ciencia y la tecnología nanoestructurada se convirtieran en una entidad definible en la pasada década, sólo se ha convertido en un campo de empeño coherente a través de la confluencia de tres líneas tecnológicas importantes:

1. Un nuevo y mejor control del tamaño y manipulación en la nanoescala de los bloques constructivos fundamentales.

2. Una nueva y mejor caracterización (resolución espacial, sensibilidad química) de materiales en la nanoescala.

3. Un nuevo y mejor conocimiento de la relación entre nanoestructura y propiedades y cómo éstas pueden ser sintonizadas ingenierilmente.

La indivisibilidad de nanociencia y nanotecnología es una realidad históricamente condicionada. En el pasado reciente, las investigaciones en nanociencia no terminaban en nanotecnología, porque no existían las condiciones para ello en la sociedad. Hoy, estas condiciones existen o se están creando a un paso muy veloz. Es este análisis el que conduce a afirmar que en el contexto actual, con el avance científico y tecnológico alcanzado, con las interrelaciones globales de las organizaciones de investigación y de socialización de los resultados, se vuelve difícil, si no imposible, separar a las nano en ciencia y tecnología y por tanto esta realidad justifica su denominación como tecnociencia.

La creciente multidisciplinariedad de las nanos es otro factor que se agrega a los anteriores para reafirmar su indivisibilidad. En un reciente artículo este carácter multidisciplinario de las nano es analizado. ${ }^{25}$ En el estudio, los autores enumeran las macrodisciplinas que contribuyen significativamente a las nanotecnologías (tabla 2). La

TABLA 2. Porciento de artículos nano en el período enero-julio del 2008 publicados en revistas científicas, clasificados por macrodisciplinas. Los porcientos no suman 100 porque un artículo puede aparecer por varias áreas. Tabla modificada de "Where does nanotechnology belong in the map of science ?" A. L. Porter y J. Youtie. Nature Nanotechnology, vol. 4, 2009.

\begin{tabular}{|l|c|c|}
\hline \multicolumn{1}{|c|}{ Macrodisciplina } & $\begin{array}{c}\text { \% de artículos nano en esta } \\
\text { macrodisciplina }\end{array}$ & $\begin{array}{c}\text { \% de artículos nano citados } \\
\text { en esta macrodisciplina }\end{array}$ \\
\hline Ciencia de Materiales & 50 & 85 \\
\hline Química & 44 & 83 \\
\hline Física & 11 & 57 \\
\hline Ciencias biomédicas & 13 & 88 \\
\hline Ciencias ingenieras & 7 & 51 \\
\hline Ciencias de la computación & 3 & 21 \\
\hline Ciencia y tecnología del & 2 & 11 \\
\hline ambiente & 1 & 9 \\
\hline Ciencias agrícolas & 1 & 6 \\
\hline Geociencias & & \\
\hline
\end{tabular}

\footnotetext{
${ }^{25}$ Porter, A. L. y J. Youtie. 2009. Where does nanotechnology belong in the map of science? Nature Nanotechnology, vol. 4.
} 
ciencia de materiales, una disciplina esencialmente tecnocientífica, clasifica asociada al $50 \%$ de las publicaciones en nano y estas publicaciones son, además, responsables del $85 \%$ de todas las citas que se realizan en el tema. De las 8 disciplinas que contribuyen a las nanotecnologías, 7 son esencialmente tecnológicas, o esconden dentro de ellas una buena parte que también es tecnología, como la física y la química. Mas aún, de las 171 áreas en las que el Science Citation Index ${ }^{26}$ clasifica los tópicos científicos, de más de 6,000 revistas descubren que en 151 de ellas se reportan artículos nanos. 52 áreas temáticas tienen más de mil artículos nano publicados en un periodo de seis meses.

Ya en un estudio realizado por el Consejo Nacional de Ciencia y Tecnología de los EUA hace diez años, esta realidad se reflejaba de manera clara al apuntarse:

Existen dos descubrimientos importantes de este estudio:

Primero, está particularmente claro que ahora somos capaces de nanoestructurar materiales para un desempeño novedoso. Ése es el tema esencial de este campo: comportamiento novedoso a través de nanoestructuración. [...]

Segundo, existe una amplia gama de disciplinas contribuyendo al desarrollo en ciencia y tecnología nanoestructurada por todo el mundo. El incremento acelerado del nivel de interdisciplinaridad en la nanoestructuración es exitante y creciente en importancia, y la intersección entre varias disciplinas es donde radica lo novedoso de su actividad. ${ }^{27}$

Separar artificialmente en lo nano ciencia de tecnología puede traer consecuencias desagradables a corto, mediano y largo plazo.

Como recientemente ha descubierto la oficina de propiedad industrial de Brasil, ${ }^{28}$ la inversión en ciencias, aun si es multimillonaria, no garantiza la apropiación de la tecnología creada o en ciernes. Miguel Jorge, presidente del Instituto Nacional de Propiedad Industrial de ese país, ha expresado que "el número de patentes brasileñas presentadas en el exterior resulta muy bajo y no se presenta una expectativa de crecimiento en los próximos años". Estamos hablando del país de América Latina que, con mucho, ha invertido más en ciencia en las últimas décadas y quien tiene el entramado científico más completo de la región. Brasil es el único país de América Latina con capacidad instrumental y facilidades científicas nacionales suficientemente actualizadas para plantearse un desarrollo autónomo en nanotecnología. ${ }^{29}$ El país invierte alrededor de 7 millones de USD sólo en tres laboratorios considerados estratégicos, y a dos laboratorios nacionales de manera adicional le dedican 6 millones per cápita anuales. Sumando los distintos financiamientos la inversión pública total del Ministerio de Ciencia y Tecnología de Brasil fue de 57 millones de USD en el año 2008 y debe mantenerse para el 2009.30

\footnotetext{
${ }^{26}$ Science Citation Index es el indicador de publicaciones científicas más reconocido internacionalmente.

${ }^{27}$ Nanostructure science and technology. A worldwide study. Resumen Ejecutivo. National Science and Technology Council (NSTC).

${ }^{28}$ Funcionarios de Brasil "perplejos" porque patentes no son promotores del comercio e innovación tecnológica. Observatorio Sudamericano de Patentes. Rebelion, <http://www.rebelion.org/noticia.php?id=88191>, 2009.

${ }^{29}$ Delgado Ramos, Gian C. 2008. "Entre la competencia y la dependencia tecnológica: la nanotecnología en el continente americano". Nómadas. Revista Crítica de Ciencias Sociales y Jurídicas, v. 17, Madrid: 265-290.

30 "Brasil investiga en grande la nanotecnología". El Mensaje. <http://www.mineducacion.gov.co/cvn/1665/ article-157906Z, 2008.
} 
Al mismo tiempo que el país celebra el crecimiento constante de su producción científica, que en 2007 alcanzó un $2.02 \%$ de los artículos publicados internacionalmente, también tiene que lamentarse de representar sólo el $0.06 \%$ del número de patentes registradas en el mundo [...] Por un lado, en Brasil, el desarrollo del conocimiento parece ir a toda máquina, obteniendo un lugar destacado entre los países de América Latina y asemejándose a países europeos, como Suiza (1.89\%), Suecia (1.81\%), Holanda (2.55\%) y Rusia (2.66\%). Pero, por otro, la producción tecnológica aún parece dejar mucho que desear en comparación con la de países como Corea del Sur (un 0.79\%), Italia (un 1.31\%), Francia (un 2.96\%) y Japón (un $22.67 \%)^{31}$

Lo paradójico es que el planteamiento nano del gobierno de Brasil se ha diseñado vinculado a la producción industrial y a la competitividad económica. A pesar de ello, esta realidad tiene como corolario, que muchas de las investigaciones llevadas a cabo en instituciones brasileñas han terminado siendo, con alta probabilidad, tecnología en otros países del mundo.

La penetración de los desarrollos nano en las regiones pobres del mundo, por parte de los centros hegemónicos, tiene varias avenidas de entrada.

En los últimos años, han proliferado eventos científicos y comisiones de búsquedas organizadas desde el norte rico para analizar el impacto de las nanotecnologías en el desarrollo. En ese contexto visitan los países subdesarrollados para evaluar sus iniciativas nano y proponer caminos de colaboración "desinteresados". Tales acciones permiten, además, conocer los actores individuales más prometedores en los distintos países. La extensión de los financiamientos, más allá de sus fronteras a investigaciones básicas, por parte de las agencias nacionales de ciencias de EUA y Europa, es un fenómeno nuevo, que tiende a crecer en áreas como la nano.

El interés manifiesto de instituciones del primer mundo en participar en los emprendimientos nanos de nuestras regiones, o de "sumar" a nuestros investigadores, e incluso laboratorios básicos, a sus proyectos en las etapas que ellos llaman precompetitivas es una realidad demostrable. A pesar de que la mayoría del tiempo esta labor se realiza de manera silenciosa, sin manifestar sus propósitos más allá de lo altruista, de vez en cuando algunos de sus artífices son más sinceros, Paul J. Herer en el reporte "Nanostructure science and technology: A worldwide study", elaborado por el Consejo Nacional de la Ciencia y la Tecnología de los Estados Unidos (WTEC) a pedido de la oficina del presidente de ese país aclara:

La información, sobre los desarrollos científicos e ingenieriles que están ocurriendo en los laboratorios alrededor del mundo es crítica para mantener la fortaleza económica y tecnológica de los Estados Unidos

$[\ldots]$

El propósito de las actividades del NSTC/WTEC es valorar los esfuerzos de I+D en otros países, áreas específicas de la tecnología, comparar estos esfuerzos y sus resultados con las investigaciones en EUA en las mismas áreas, e identificar oportunidades para la colaboración internacional y la investigación precompetitiva

Está claro quien se lleva y se llevará la tajada del león en esta colaboración "precompetitiva".

En ocasiones, separar lo básico de lo tecnológico de manera forzada se usa como argumento para facilitar tales penetraciones tanto a nivel colectivo como individual.

\footnotetext{
31 "Brasil sufre por la separación entre la universidad y la empresa". <http://www.universia.edu.uy/index. php?option=com_content \&task=view\&id=5866>. 2008.
} 
Ahora bien, que asumamos lo nano como un quehacer tecnocientífico no puede conducirnos a querer absolutizar sólo un aspecto de la relación entre conocimiento e innovación. Como señala Nuñez Jover "el término tecnociencia no necesariamente conduce a cancelar las identidades de la ciencia y la tecnología".

Reducir todo a tecnología, minimizando las otras funciones sociales que cumple la ciencia tiene sentido en un sistema social capitalista que ve al científico y a la ciencia como meros catalizadores e incrementadores de su cuota de ganancia. El científico es un "obrero" altamente calificado y la tecnociencia es una herramienta maximizadora de las fuerzas productivas y a la vez de control ideológico y real sobre la sociedad. Las nano entran de lleno en esta lógica.

El Prof. Alex Callinicos, a quien ya nos hemos referido, ha analizado las consecuencias de la penetración neoliberal en las universidades británicas

las consecuencias fueron calamitosas. A los académicos y a otros miembros del personal universitario se les niega cada vez más la oportunidad de buscar el conocimiento por sí mismos y la oportunidad de atender las necesidades educativas y de otro tipo de los estudiantes. [...] Los estudiantes [...] son también víctimas de la subordinación de las universidades a las prioridades del mercado: El neoliberalismo en la educación superior significa que esta lógica de la competencia se interioriza profundamente en la manera de funcionar de las universidades.

Este cambio en la función social de las universidades se hace de manera explícita:

las universidades deberán convertirse no sólo en centros de enseñanza e investigación, sino en ejes de las redes de innovación en las economías locales, ayudando a lanzar empresas desde las universidades, por ejemplo. Las universidades deberían de ser minas a cielo abierto de la economía del conocimiento. ${ }^{32}$

Como consecuencia de ello, los recursos a los centros de educación superior se asignan casi exclusivamente sobre la base de su aporte a la competitividad económica. Los gobiernos del primer mundo establecen esquemas de clasificación y financiamiento para sus universidades de acuerdo con esas líneas.

Reducir las investigaciones exclusivamente a su aspecto tecnológico en las ciencias como las nano es ahogar sus otras dimensiones igualmente imprescindibles a una sociedad.

Deberíamos defender lo que es valioso en las universidades existentes, contra la amenaza de destruir lo que representan por medio de la transformación neoliberal. Por ejemplo [...], es importante que las universidades sigan ofreciendo un espacio intelectual en el que se desarrolle el pensamiento crítico.

Debe ser una preocupación que estas "otras" actividades, que son también inherentes a la investigación científica natural, tengan espacio y recursos, garantizándoles un estado de salud adecuado que permita su crecimiento indetenido y su impacto efectivo en la sociedad.

La sociedad tecnocientífica absoluta es una pesadilla futurista condenada de antemano al fracaso; esta afirmación, que bien puede asimilarse de manera inmediata, es

\footnotetext{
${ }^{32}$ Leadbeater. C. 2009. Living on thin air. Viking: 114.
} 
en muchos casos mal comprendida a la hora de establecer políticas y tomar decisiones. La crisis actual de la contaminación ambiental es un buen ejemplo de hacia dónde nos puede conducir la absolutización tecnológica, la minimización de la necesidad del conocimiento básico y la matriz explosiva de la ambición humana.

En la época de la convergencia de las ciencias es difícil prever qué área del conocimiento mañana será relevante para la sustentabilidad de otras. Ése es el contexto tecnocientífico actual y no se puede eludir.

\title{
PORQUE YA VA SIENDO MUY LARGO EL DISCURSO ARGUMENTAL SE CONCLUYE PRETENDIENDO SINTETIZAR ALGUNAS IDEAS
}

¿Nanociencia o nanotecnología? es una pregunta que permite abordar un conjunto de problemas asociados a la revolución nano en el contexto actual. Como se ha pretendido argumentar, su respuesta, en términos de tecnociencia, no agota la disyuntiva y más bien conduce a realizar un enfoque del tema que rebase lo meramente tecnológico o científico para adentrase en cuestiones sociales, económicas y políticas fundamentales que no pueden eludirse.

Una nanociencia que pretenda no estar unida de manera orgánica a un desarrollo nanotecnológico está condenada a abortar en un plazo no muy largo de tiempo. Esa realidad no es exclusiva de la región. Como afirma Richard W. Siegel, del Consejo Nacional de las Ciencias y las Tecnologías de los EUA:

\begin{abstract}
Entre los retos que tenemos por delante están aquellos vinculados con realizar los avances necesarios en las tecnologías habilitantes que nos permitan un progreso rápido y continuo en este campo. Debemos aumentar nuestras capacidades de caracterización y visualización y análisis químico a escalas cada vez más pequeñas, y, eventualmente, incorporar recursos computacionales que nos permitan dirigirlas, si es que realmente queremos aprovechar totalmente las oportunidades.
\end{abstract}

En este empeño son muchas las amenazas externas que acechan. Ninguna iniciativa nano se desarrolla aislada en el mundo, sino que tiene en su universo de existencia en una competencia desenfrenada. En este contexto, buscar de manera inteligente alianzas estratégicas o tácticas es esencial, del mismo modo que lo es reconocer de manera rápida los peligros provenientes de otros actores.

En una estrategia nano no puede subestimarse el factor tiempo. La celeridad en el desarrollo de esta área y su creciente impacto hacen que cada día se cierren puertas y no se alcancen oportunidades. No llegar a tiempo significa en la práctica no llegar. Esa realidad obliga a los países pobres a aprovechar al máximo el margen de oportunidad que se les brinda - por estrecho que sea- para aprovechar los desarrollos alcanzados y potenciarlos.

Por otro lado, intentar absolutizar un sólo aspecto de la tecnociencia, en el caso de lo nano, trae el peligro de no ver las otras dimensiones del mismo fenómeno. Dimensiones que alcanzan tanto lo científico como lo social o lo ecológico. Absolutizar el aspecto tecnológico puede traer a la realidad la potencial pesadilla de un futuro disfuncional e inviable.

Con las nano, el futuro es incierto para cualquier proyecto de investigación. No está claro qué desarrollo conducirá a una innovación exitosa. Si bien esto es cierto para casi todas las ramas de las ciencias en algún que otro grado, esta realidad es 
particularmente inevitable en las nano en su estado actual. Investigar e innovar pasa necesariamente por tomar decisiones arriesgadas, los países del primer mundo minimizan globalmente los riesgos al poder apoyar en mayor o menor medida toda la gama de posibilidades que se van abriendo, pero aun ellos necesitan de la colaboración internacional para lograr conjurar los peligros. Para los países pobres este reto es aún más gigantesco y su solución pasa por la integración (como manera más cabal de cooperación) de esfuerzos a nivel supranacional. La búsqueda de la integración con sujetos que comparten los mismos objetivos y la misma suerte, en caso de fracasar, es esencial para poder hacer frente a la invasión nano que nos viene del primer mundo.

A la hora de plantearse buscar solución a nuevos retos como el que nos plantean las nano, quizás valga citar lo que recientemente afirmara Alfredo Guevara: "La verdad no es absoluta. La verdad es siempre una aproximación, constantemente cambiante (...). El problema es que el cambio no puede ser un acto frívolo, pero el cambio es necesario constantemente. Ante nuevas situaciones, nuevas soluciones." ${ }^{33}$

La revolución nano nos pone al día el reto de cambiar nuestra forma de abordar los fenómenos tecnocientíficos, hacerlo es un imperativo si no queremos fracasar en su implicación social, económica y política, hasta llegar a naufragar en su misma esencia de ciencia.

\footnotetext{
${ }^{33}$ El peor enemigo de la Revolución es la ignorancia. Alfredo Guevara entrevista de Leandro Estupiñan. http:// www.revistacaliban.cu/entrevista.php?numero=5, 2009
} 